\title{
Circulating Autoantibodies to LGALS3BP: A Novel Biomarker for Cancer
}

\author{
Antonino Grassadonia, ${ }^{1}$ Nicola Tinari, ${ }^{1,2}$ Clara Natoli, ${ }^{1}$ \\ Galit Yahalom, ${ }^{3}$ and Stefano Iacobelli ${ }^{1,2}$ \\ ${ }^{1}$ Department of Experimental and Clinical Sciences, “G. D’Annunzio” University, 66100 Chieti, Italy \\ ${ }^{2}$ MediaPharma s.r.l., Via dei Vestini 31, 66100 Chieti, Italy \\ ${ }^{3}$ Eventus Diagnostics, Inc., Jehuda Hills, 90880 Ora, Israel
}

Correspondence should be addressed to Antonino Grassadonia; grassa@unich.it

Received 8 July 2013; Revised 11 October 2013; Accepted 11 October 2013

Academic Editor: Grant Izmirlian

Copyright (C) 2013 Antonino Grassadonia et al. This is an open access article distributed under the Creative Commons Attribution License, which permits unrestricted use, distribution, and reproduction in any medium, provided the original work is properly cited.

\begin{abstract}
Purpose. Circulating autoantibodies have been extensively investigated as possible markers for early diagnosis of cancer. The present study was carried out to investigate whether anti-LGALS3BP IgG autoantibodies could be classified as a biomarker for malignant tumors. Methods. An in-house developed enzyme-linked immunosorbent assay was used to detect autoantibodies to LGALS3BP in sera from 71 patients with various types of cancers and 54 healthy subjects matched by age and gender. Results. Patients with cancer have significant higher circulating levels of anti-LGALS3BP antibodies as compared to control subjects $(P<0.001)$. The test has a sensitivity of $33 \%$ and a specificity of $98 \%$. Conclusions. Anti-LGALS3BP IgG autoantibodies are a promising biomarker for malignant tumors and could play a role in the development of a multimarker assay for the early detection of cancer.
\end{abstract}

\section{Introduction}

Cancer is the second leading cause of death worldwide, accounting for about 600,000 deaths in the United States in 2012 [1]. Despite significant improvements in treatment, early detection remains the most important prognostic factor predicting of better outcome [2-4]. Current cancer screening methods, including mammography for breast cancer, colonoscopy for colon cancer, computed tomography for lung cancer, prostate-specific antigen for prostate cancer, and Papanicolaou stains for cervical cancer, have demonstrated some limitations in terms of sensitivity, specificity, complexity, cost, and compliance [5].

Serum tumor-associated antigens (TAAs) have been extensively studied for early cancer detection because of the simplicity and reliability of the tests used for their determination, such as western blot and enzyme-linked immunosorbent assay (ELISA). Unfortunately, they are transiently secreted and rapidly eliminated from blood circulation [6, 7] and usually reach a detectable concentration only in advanced stage of the disease [8].
Along with TAAs, autoantibodies are frequently detected in sera from patients affected by different types of neoplasms [9]. This finding has been interpreted as an attempt of the immune system to block invasion and spreading of cancer cells in the organism. Circulating autoantibodies have biological and biochemical characteristics that render them particularly suitable to screen subjects at cancer risk. In fact, they may develop early in the process of tumorigenesis, when premalignant or malignant cells begin to express altered molecules as a result of cell transformation [10, 11]. In addition, they can easily be detected in the serum because of the usual high concentration and long-time stability [9]. For these reasons, great efforts have been made in recent years to identify circulating autoantibodies directed against cancer-related proteins in order to build up tests for the early detection of neoplastic disease [12-15].

In this study, we investigated the production of autoantibodies against lectin galactoside-binding soluble 3 binding protein (LGALS3BP) in patients affected by different types of cancer. LGALS3BP, also known as 90K or Mac-2 BP [16], has been largely regarded as a TAA, since it is present at elevated 
TABle 1: Patient characteristics.

\begin{tabular}{|c|c|c|c|c|c|}
\hline & $N$ & Median age (years) & Range (years) & Males & Females \\
\hline Controls & 54 & 41 & $26-58$ & 34 & 20 \\
\hline Cancers & 71 & 64 & $27-87$ & 38 & 33 \\
\hline GI & 15 & 63 & $44-87$ & 10 & 5 \\
\hline NSCLC & 13 & 67 & $55-77$ & 11 & 2 \\
\hline $\mathrm{BC}$ & 12 & 62 & $33-77$ & 1 & 11 \\
\hline NET & 10 & 61.5 & $39-75$ & 7 & 3 \\
\hline UG & 10 & 61.5 & $40-80$ & 4 & 6 \\
\hline M & 7 & 52 & $35-60$ & 3 & 4 \\
\hline Others & 4 & 47.5 & $27-77$ & 2 & 2 \\
\hline
\end{tabular}

GI: gastrointestinal cancer; NSCLC: non-small-cell lung cancer; BC: breast cancer; NET: neuroendocrine tumors; UG: urogenital tract cancer; M: melanoma.

concentrations in the blood of cancer patients and is overexpressed in the vast majority of cancer tissues [17]. Both high serum and tumor levels of LGALS3BP have been associated with a poor outcome in patients with different types of neoplasms [18-21]. LGALS3BP has also been implicated in cancer progression by modulating tumor cell adhesion processes. In fact, it can bind important molecules associated with the membrane of tumor cells, such as galectin-3, galectin-1, and $\beta 1$-integrins $[16,22,23]$. Additionally, LGALS3BP can interact with extracellular matrix proteins such as collagen, fibronectin, and laminin [23, 24].

Here we show that patients with different types of cancer, but not healthy subjects, develop autoantibodies against LGALS3BP. This finding discloses the capability of LGALS3BP to trigger a humoral immune response in cancer patients and provides the basis for further investigation on a possible use of anti-LGALS3BP antibodies as biomarkers for early diagnosis of cancer.

\section{Materials and Methods}

2.1. Patients. The study population consisted of 71 patients with different types of cancers, 15 gastrointestinal cancers, 13 non-small-cell lung cancers (NSCLC), 12 breast cancers, 10 neuroendocrine tumors (NET), 10 urogenital tract cancers, 7 melanomas, and 4 others (2 gliomas, 1 tongue cancer, and 1 osteosarcoma). All patients were in stage IV disease. Fiftyfour healthy donors were also included as controls. Patients characteristics are summarized in Table 1.

All blood samples were obtained with donor's consent. Serum was collected from total blood and stored at $-20^{\circ} \mathrm{C}$ after adding $0.01 \%$ sodium azide.

2.2. Human Recombinant LGALS3BP. Human recombinant LGALS3BP was immunoaffinity-purified from serum-free supernatant of human embryonic kidney EBNA-293 cells (Invitrogen, Carlsbad, CA, USA) transfected with LGALS3BP cDNA [23].

2.3. Measurement of LGALS3BP Autoantibodies. LGALS3BP autoantibodies were determined by enzyme-linked immunosorbent assay. Ninety-six well microtiter plates (Nalge Nunc, Denmark) were coated with recombinant purified
LGALS3BP protein $\left(5 \mu \mathrm{g} / \mathrm{mL}\right.$ in PBS) at $4^{\circ} \mathrm{C}$ overnight. The plate was saturated with $1 \%$ BSA and $0.05 \%$ Tween-20 in PBS (saturation buffer) at $37^{\circ} \mathrm{C}$ for $2 \mathrm{~h}$ and then incubated with $100 \mu \mathrm{L}$ of serum from healthy donors or patients at $37^{\circ} \mathrm{C}$ for $1 \mathrm{~h}$. The serum was diluted 1:100 in saturation buffer. After 3 washes with $0.05 \%$ Tween-20 in PBS (washing buffer), a second incubation was performed with $100 \mu \mathrm{L}$ of biotinconjugated anti-human IgG (Sigma, St Louis, Mo, USA), diluted 1:2000 in saturation buffer, at $37^{\circ} \mathrm{C}$ for $1 \mathrm{~h}$. After washing, a third incubation was performed with peroxidaseconjugated ExtrAvidin (Sigma, St Louis, Mo, USA) diluted $1: 4000$ in saturation buffer, at $37^{\circ} \mathrm{C}$ for $45 \mathrm{~min}$. After washing, $100 \mu \mathrm{L}$ of TMB substrate was added to each well and the plate was shaken at room temperature for $15 \mathrm{~min}$. Eventually, the reaction was stopped by adding $100 \mu \mathrm{L}$ of $1 \mathrm{M} \mathrm{H}_{2} \mathrm{SO}_{4} /$ well and color revealed by reading absorbance at $450 \mathrm{~nm}$ in an automatic ELISA reader.

2.4. Measurement of LGALS3BP. To measure serum concentration of LGALS3BP a commercially available ELISA kit (Diesse, Siena, Italy) was used according to the manufacturer's instructions. Results were expressed in $\mu \mathrm{g} / \mathrm{mL}$.

2.5. Western Blot Analyses. Purified human recombinant LGALS3BP $(10 \mu \mathrm{g} /$ well $)$ was separated by $8 \%$ SDS-PAGE under reducing conditions and transferred to nitrocellulose using standard procedures. Membrane was saturated in blocking buffer phosphate buffered saline with $0.05 \%$ Tween20 (PBS-T), 5\% low-fat milk, $1 \%$ BSA and at $4^{\circ} \mathrm{C}$ overnight. After washing in PBS-T, membrane was incubated with serum from controls or patients affected by cancer, diluted in an equal volume with PBS-T, at room temperature for $1 \mathrm{~h}$. After washing in PBS-T, membrane was incubated with biotinylated anti-human IgG (Sigma, St Louis, Mo, USA) diluted 1:1000 in blocking buffer at room temperature for $1 \mathrm{~h}$ and then with ExtrAvidin Peroxidase (Sigma, St Louis, Mo, USA) 1:500 in Blocking Buffer for $30 \mathrm{~min}$. To identify the presence of antibodies bound to LGALS3BP, the colorimetric substrate $\mathrm{DAB}\left(3,3^{\prime}\right.$-diaminobenzidine) was added to the membrane for $10 \mathrm{~min}$.

2.6. Statistical Analyses. Each sample was assayed in triplicate and the mean value was used for statistical analyses. 


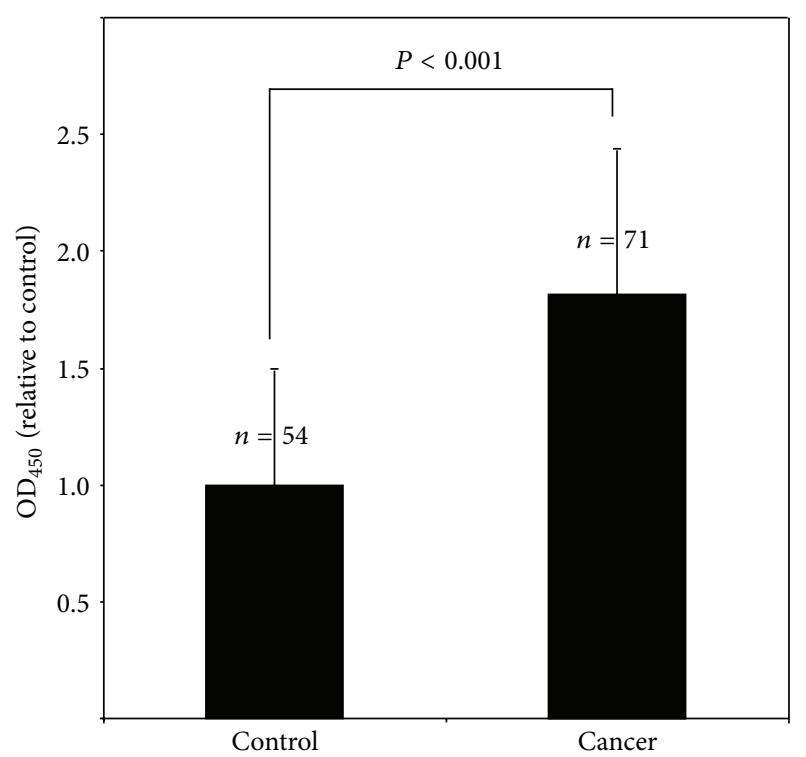

(a)

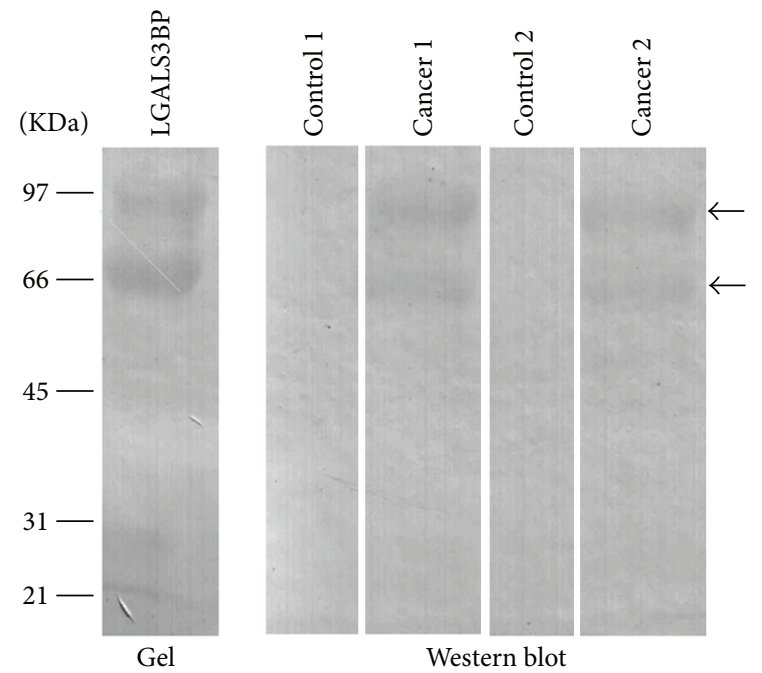

(b)

FIGURE 1: Identification of circulating anti-LGALS3BP autoantibodies in the blood of cancer patients. (a) Western blot performed under reducing conditions. Purified LGALS3BP was separated by SDS-PAGE and transferred to nitrocellulose membrane. Gel stained in Coomassie brilliant blue detected two bands at about $97 \mathrm{KDa}$ and $66 \mathrm{KDa}$, corresponding to the full length and cleaved form of the protein, respectively. Membrane was incubated with serum from healthy donors (controls) or patients affected by cancer and then processed in order to identify IgG antibodies. Membrane exposed to serum from patients, but not from controls, developed bands at the same size of LGALS3BP (arrows). (b) Quantification of serum LGALS3BP in healthy donors and cancer patients by ELISA. The bars represent the mean of 3 different assays in triplicate \pm SD.

TABLE 2: Measurement of circulating autoantibodies in the different groups of cancer patients.

\begin{tabular}{|c|c|c|c|c|c|}
\hline & $N$ & OD $($ Mean \pm SD $)$ & $P^{*}$ & Number of patients over cut-off limit (\%) & $P^{* *}$ \\
\hline Controls & 54 & $0.5 \pm 0.25$ & & $1 / 54(2)$ & \\
\hline Cancers & 71 & $0.89 \pm 0.44$ & $<0.001$ & $26 / 71(37)$ & $<0.001$ \\
\hline GI & 15 & $0.97 \pm 0.50$ & 0.023 & $5 / 15(33)$ & 0.001 \\
\hline NSCLC & 13 & $1.09 \pm 0.65$ & 0.013 & $6 / 13(46)$ & $<0.001$ \\
\hline $\mathrm{BC}$ & 12 & $0.70 \pm 0.45$ & NS & $3 / 12(25)$ & 0.017 \\
\hline NET & 10 & $0.92 \pm 0.22$ & $<0.001$ & $5 / 10(50)$ & $<0.001$ \\
\hline UG & 10 & $1.05 \pm 0.60$ & 0.001 & $5 / 10(50)$ & $<0.001$ \\
\hline M & 7 & $0.54 \pm 0.30$ & NS & $1 / 7(14)$ & NS \\
\hline Others & 4 & $0.69 \pm 0.50$ & NS & $1 / 4(25)$ & NS \\
\hline
\end{tabular}

GI: gastrointestinal cancer; NSCLC: non-small-cell lung cancer; BC: breast cancer; NET: neuroendocrine tumors; UG: urogenital tract cancer; M: melanoma.

*Mann-Whitney $U$ test; ${ }^{* *}$ Fisher's exact test; NS: not significant.

The results were expressed as mean \pm standard deviation (SD). Differences between groups were tested with the nonparametric Mann-Whitney $U$ test. The normal upper cut-off value of anti-LGALS3BP antibody in sera was set at the value of the mean $+2 S D$ of the absorbance in 54 healthy donors. Differences in the proportion over the cut-off limit were evaluated by Fisher's exact test. Correlations were estimated using the Spearman rank-correlation coefficient $\left(r_{s}\right)$.

\section{Results}

Patients affected by different types of cancers showed significant increased levels of LGALS3BP autoantibodies
$(P<0.001)$ compared to healthy subjects (Figure 1(a)). The specific binding of antibodies to LGALS3BP was confirmed in western blot, where the presence of anti-LGALS3BP IgG was detected as two bands at about $97 \mathrm{KDa}$ and $66 \mathrm{KDa}$, the exact size of the protein in its full length and cleaved form, respectively (Figure 1(b)). Among patients, NSCLC, gastrointestinal cancer, urogenital tract cancer, and NET reached the highest levels of autoantibodies, while there was no significant increase in breast cancer and melanoma (Table 2).

Setting the normal upper cut-off limit of ELISA at OD 0.99 (the mean $+2 \mathrm{SD}$ of the absorbance in sera from healthy individuals), the assay showed a sensitivity of 33\% (26/71 
TABLE 3: Serum levels of LGALS3BP.

\begin{tabular}{lcc}
\hline & $N$ & $\mu \mathrm{g} / \mathrm{mL}($ Mean $\pm \mathrm{SD})$ \\
\hline Controls & 54 & $6.36 \pm 1.16$ \\
Cancers & 71 & $13.19 \pm 2.37$ \\
GI & 15 & $16.68 \pm 0.95$ \\
NSCLC & 13 & $12.17 \pm 0.75$ \\
BC & 12 & $14.07 \pm 1.08$ \\
NET & 10 & $10.28 \pm 1.25$ \\
UG & 10 & $13.13 \pm 1.2$ \\
M & 7 & $11.13 \pm 0.66$ \\
Others & 4 & $11.73 \pm 0.93$ \\
\hline
\end{tabular}

GI: gastrointestinal cancer; NSCLC: non-small-cell lung cancer; BC: breast cancer; NET: neuroendocrine tumors; UG: urogenital tract cancer; M: melanoma.

patients were positive) and a specificity of $98 \%$ (only 1 out of 54 controls was positive). All cancer groups, but melanoma, showed autoantibody levels significantly above the cut-off limit (Table 2).

Serum levels of LGALS3BP were also determined. As expected, the protein was significantly higher in patients with cancer compared to normal subjects ( 13.19 versus $6.36 \mu \mathrm{g} / \mathrm{mL}$, $P<0.001$ ) (Table 3), but values did not correlate with the levels of autoantibodies (data not shown).

\section{Discussion}

Using ELISA technique, we show that LGALS3BP is able to elicit host immune response with IgG autoantibodies production in patients affected by different types of cancer. Anti-LGALS3BP IgG concentrations were higher in patients with NSCLC, gastrointestinal cancer, urogenital tract cancer, and NET than in those with breast cancer and melanoma, but the number of patients in each subgroup was insufficient to yield a statistically reliable comparison.

It is generally accepted that tumor proteins perceived as nonself by the immune system and able to trigger an immune response are often overexpressed [25], mutated [26], misfolded [27], or endowed with posttranslational changes, such as alterations of glycosylation and phosporylation [28, 29]. Consistently, LGALS3BP may evoke autoantibody production because it is overexpressed in cancer cells, and also because it may carry posttranslational alterations in its glycidic moiety. Qualitative and quantitative changes in $\mathrm{O}-$ and $\mathrm{N}$-glycosylation of proteins are frequent events in malignancies $[30,31]$ and differences in the glycosylation pattern of LGALS3BP have been reported in some cancer cell lines [24].

Finalistically, antitumor directed antibodies are generated in order to halt tumor initiation and progression. As this process initiates early in cancerogenesis, in a preclinical phase of the disease, autoantibodies production has been considered a useful biomarker for early cancer diagnosis [32-35]. In this study, the serum levels of anti-LGALS3BP IgG detected in cancer patients were not correlated with those of LGALS3BP, indicating that even small amount of the protein, as expected in the initial phase of cancer growth, may generate high concentrations of autoantibodies. This evidence suggests a possible role for anti-LGALS3BP IgG in the early detection of cancer.

Although it is not possible to exclude that the presence of autoantibodies might affect the correct quantification of LGALS3BP by ELISA, the identification of anti-LGALS3BP $\mathrm{IgG}$ in western blot indicates that the epitopes recognized by these autoantibodies are different from those recognized by the antibody used in ELISA. In fact, western blot performed under reducing conditions can detect only autoantibodies directed to epitopes expressed on the primary structure of LGALS3BP, while the monoclonal antibody contained in the commercially available ELISA kit, known as SP2, recognizes a conformational epitope shaped in the native form of the protein and, for this reason, is not suitable for western blotting.

In cancer patients, autoantibodies are frequently directed against cellular proteins that play key roles in tumor progression, including molecules involved in cell cycle, signal transduction, proliferation, and apoptosis [36-38]. As a consequence, the identification of the molecular target of autoantibodies might be of relevance in designing new antitumor agents. We can, therefore, speculate that LGALS3BP could be a candidate for targeted therapies against cancer.

In the past few years, the growing interest in autoantibodies as a possible tool for the early diagnosis of cancer and the identification of new targets for molecular therapy has made the development of high-throughput techniques such as SEREX (serological analysis of tumor antigens by recombinant cDNA expression cloning), phage display, protein microarray, SERPA (serological proteome analysis), and MAPPing (multiple affinity protein profiling) [39] able to detect simultaneously multiple autoantibodies and their cognate TAAs. With these methods, several new targets have been identified, but collectively single antigens have shown low sensitivity and specificity to be used in clinical screening [40]. To increase sensitivity, autoantibody diagnostic tests combining two or more TAAs [41-43] or evaluating wellknown biomarkers in combination with autoantibodies [44] have been developed. For example, a large screening study of high-risk individuals for lung cancer has validated a test measuring autoantibody levels against a panel of six TAAs (p53, NY-ESO-1, CAGE, GBU4-5, Annexin 1, and SOX2) [41]. In another study, the combination of p53 autoantibodies and CA125 levels increased sensitivity for ovarian cancer from $73.8 \%$ (CA125) to $85.7 \%$ (CA 125 plus p53 autoantibodies) [44]. The determination of anti-LGALS3BP IgG presented in this study showed a very high specificity (98\%), but a low sensitivity (33\%), comparable to that reported for autoantibodies against single TAA, ranging between $10 \%$ and $30 \%$ [40]. Therefore, our anti-LGALS3BP ELISA lacks sufficient sensitivity to be used in early cancer diagnosis. Nevertheless, the determination of autoantibodies against LGALS3BP might be useful to increase the sensitivity of tests combining multiple autoantibodies. Preliminary results indicate that using a set of different autoantibodies combined with autoantibodies for LGALS3BP will increase the sensitivity for breast cancer patients to $50 \%$ and maintain the high specificity (98\%) [45]. These preliminary results should be tested for other types of cancers as well. 
In summary, our study demonstrated the presence of autoantibodies against LGALS3BP in the serum of patients with different types of cancers. These autoantibodies may be used in developing screening tests for early-stage cancer detection.

\section{References}

[1] R. Siegel, D. Naishadham, and A. Jemal, "Cancer statistics, 2013," CA Cancer Journal for Clinicians, vol. 63, no. 1, pp. 11-30, 2013.

[2] F. A. Coumans, S. Siesling, and L. W. Terstappen, "Detection of cancer before distant metastasis," BMC Cancer, vol. 13, no. 1, article 283, 2013.

[3] W. S. Atkin, R. Edwards, I. Kralj-Hans et al., "Once-only flexible sigmoidoscopy screening in prevention of colorectal cancer: a multicentre randomised controlled trial," The Lancet, vol. 375, no. 9726, pp. 1624-1633, 2010.

[4] B. Levin, D. A. Lieberman, B. McFarland et al., "Screening and surveillance for the early detection of colorectal cancer and adenomatous polyps, 2008: a Joint guideline from the American Cancer Society, the US Multi-Society Task Force on Colorectal Cancer, and the American College of Radiology," Gastroenterology, vol. 134, no. 5, pp. 1570-1595, 2008.

[5] R. A. Smith, V. Cokkinides, and O. W. Brawley, "Cancer screening in the United States, 2012: a review of current American Cancer Society guidelines and current issues in cancer screening," CA Cancer Journal for Clinicians, vol. 62, no. 2, pp. 129-142, 2012.

[6] M. G. Wahrenbrock and A. Varki, "Multiple hepatic receptors cooperate to eliminate secretory mucins aberrantly entering the bloodstream: are circulating cancer mucins the "tip of the iceberg"?" Cancer Research, vol. 66, no. 4, pp. 2433-2441, 2006.

[7] H. H. Wandall, O. Blixt, M. A. Tarp et al., "Cancer biomarkers defined by autoantibody signatures to aberrant O-glycopeptide epitopes," Cancer Research, vol. 70, no. 4, pp. 1306-1313, 2010.

[8] M. J. Duffy, D. Evoy, and E. W. McDermott, "CA 15-3: uses and limitation as a biomarker for breast cancer," Clinica Chimica Acta, vol. 411, no. 23-24, pp. 1869-1874, 2010.

[9] K. S. Anderson and J. LaBaer, "The sentinel within: exploiting the immune system for cancer biomarkers," Journal of Proteome Research, vol. 4, no. 4, pp. 1123-1133, 2005.

[10] O. J. Finn, "Immune response as a biomarker for cancer detection and a lot more," The New England Journal of Medicine, vol. 353, no. 12, Article ID 058157, pp. 1288-1290, 2005.

[11] E. M. Tan, "Autoantibodies as reporters identifying aberrant cellular mechanisms in tumorigenesis," Journal of Clinical Investigation, vol. 108, no. 10, pp. 1411-1415, 2001.

[12] K. S. Anderson, N. Ramachandran, J. Wong et al., "Application of protein microarrays for multiplexed detection of antibodies to tumor antigens in breast cancer," Journal of Proteome Research, vol. 7, no. 4, pp. 1490-1499, 2008.

[13] P. J. Mintz, J. Kim, K.-A. Do et al., "Fingerprinting the circulating repertoire of antibodies from cancer patients," Nature Biotechnology, vol. 21, no. 1, pp. 57-63, 2003.

[14] S. R. Pereira-Faca, R. Kuick, E. Puravs et al., "Identification of 14-3-3 $\theta$ as an antigen that induces a humoral response in lung cancer," Cancer Research, vol. 67, no. 24, pp. 12000-12006, 2007.

[15] N. Ramachandran, J. V. Raphael, E. Hainsworth et al., "Nextgeneration high-density self-assembling functional protein arrays," Nature Methods, vol. 5, no. 6, pp. 535-538, 2008.
[16] A. Grassadonia, N. Tinari, I. Iurisci et al., "90K (Mac-2 BP) and galectins in tumor progression and metastasis," Glycoconjugate Journal, vol. 19, no. 7-9, pp. 551-556, 2002.

[17] S. Iacobelli, E. Arno, P. Sismondi et al., "Measurement of a breast cancer associated antigen detected by monoclonal antibody SP-2 in sera of cancer patients," Breast Cancer Research and Treatment, vol. 11, no. 1, pp. 19-30, 1988.

[18] S. Iacobelli, P. Sismondi, M. Giai et al., "Prognostic value of a novel circulating serum $90 \mathrm{~K}$ antigen in breast cancer," British Journal of Cancer, vol. 69, no. 1, pp. 172-176, 1994.

[19] A. G. Zeimet, C. Natoli, M. Herold et al., "Circulating immunostimulatory protein $90 \mathrm{~K}$ and soluble interleukin-2-receptor in human ovarian cancer," The International Journal of Cancer, vol. 68, no. 1, pp. 34-38, 1996.

[20] A. Marchetti, N. Tinari, F. Buttitta et al., "Expression of $90 \mathrm{~K}$ (Mac-2 BP) correlates with distant metastasis and predicts survival in stage I non-small cell lung cancer patients," Cancer Research, vol. 62, no. 9, pp. 2535-2539, 2002.

[21] N. Tinari, R. Lattanzio, P. Querzoli et al., "High expression of $90 \mathrm{~K}(\mathrm{Mac}-2 \mathrm{BP})$ is associated with poor survival in nodenegative breast cancer patients not receiving adjuvant systemic therapies," International Journal of Cancer, vol. 124, no. 2, pp. 333-338, 2009.

[22] H. Inohara, S. Akahani, K. Koths, and A. Raz, "Interactions between galectin-3 and Mac-2-binding protein mediate cellcell adhesion," Cancer Research, vol. 56, no. 19, pp. 4530-4534, 1996.

[23] T. Sasaki, C. Brakebusch, J. Engel, and R. Timpl, "Mac-2 binding protein is a cell-adhesive protein of the extracellular matrix which self-assembles into ring-like structures and binds $\beta 1$ integrins, collagens and fibronectin," The EMBO Journal, vol. 17, no. 6, pp. 1606-1613, 1998.

[24] T. A. Ulmer, V. Keeler, L. Loh et al., "Tumor-associated antigen 90K/Mac-2-binding protein: possible role in colon cancer," Journal of Cellular Biochemistry, vol. 98, no. 5, pp. 1351-1366, 2006.

[25] Y.-T. Chen, M. J. Scanlan, U. Sahin et al., "A testicular antigen aberrantly expressed in human cancers detected by autologous antibody screening," Proceedings of the National Academy of Sciences of the United States of America, vol. 94, no. 5, pp. 19141918, 1997.

[26] T. Soussi, "p53 Antibodies in the sera of patients with various types of cancer: a review," Cancer Research, vol. 60, no. 7, pp. 1777-1788, 2000.

[27] U. Schubert, L. C. Antón, J. Gibbs, C. C. Norbury, J. W. Yewdell, and J. R. Bennink, "Rapid degradation of a large fraction of newly synthesized proteins by proteasomes," Nature, vol. 404, no. 6779, pp. 770-774, 2000.

[28] P. Blume-Jensen and T. Hunter, "Oncogenic kinase signalling," Nature, vol. 411, no. 6835, pp. 355-365, 2001.

[29] S. Hakomori, "Tumor-associated carbohydrate antigens," Annual Review of Immunology, vol. 2, Article ID 040184, pp. 103-126, 1984.

[30] S.-I. Hakomori, "Tumor-associated carbohydrate antigens defining tumor malignancy: basis for development of anticancer vaccines," Advances in Experimental Medicine and Biology, vol. 491, pp. 369-402, 2001.

[31] K. O. Lloyd, "Philip Levine award lecture. Blood group antigens as markers for normal differentiation and malignant change in human tissues," American Journal of Clinical Pathology, vol. 87, no. 1, pp. 129-139, 1987. 
[32] M. Caron, G. Choquet-Kastylevsky, and R. Joubert-Caron, "Cancer immunomics using autoantibody signatures for biomarker discovery," Molecular and Cellular Proteomics, vol. 6, no. 7, pp. 1115-1122, 2007.

[33] F. M. Brichory, D. E. Misek, A.-M. Yim et al., "An immune response manifested by the common occurrence of annexins I and II autoantibodies and high circulating levels of IL-6 in lung cancer," Proceedings of the National Academy of Sciences of the United States of America, vol. 98, no. 17, pp. 9824-9829, 2001.

[34] F. Le Naour, D. E. Misek, M. C. Krause et al., "Proteomics-based identification of RS/DJ-1 as a novel circulating tumor antigen in breast cancer," Clinical Cancer Research, vol. 7, no. 11, pp. 33283335, 2001.

[35] M. J. Nam, J. Madoz-Gurpide, H. Wang et al., "Molecular profiling of the immune response in colon cancer using protein microarrays: occurrence of autoantibodies to ubiquitin Cterminal hydrolase L3," Proteomics, vol. 3, no. 11, pp. 2108-2115, 2003.

[36] Y. Hirohashi, T. Torigoe, S. Inoda et al., "The functioning antigens: beyond just as the immunological targets," Cancer Science, vol. 100, no. 5, pp. 798-806, 2009.

[37] F. F. Madrid, "Autoantibodies in breast cancer sera: candidate biomarkers and reporters of tumorigenesis," Cancer Letters, vol. 230, no. 2, pp. 187-198, 2005.

[38] M. F. Ullah and M. Aatif, "The footprints of cancer development: cancer biomarkers," Cancer Treatment Reviews, vol. 35, no. 3, pp. 193-200, 2009.

[39] H. T. Tan, J. Low, S. G. Lim, and M. C. M. Chung, "Serum autoantibodies as biomarkers for early cancer detection," The FEBS Journal, vol. 276, no. 23, pp. 6880-6904, 2009.

[40] C. A. Casiano, M. Mediavilla-Varela, and E. M. Tan, "Tumorassociated antigen arrays for the serological diagnosis of cancer," Molecular and Cellular Proteomics, vol. 5, no. 10, pp. 1745-1759, 2006.

[41] P. Boyle, C. J. Chapman, S. Holdenrieder et al., "Clinical validation of an autoantibody test for lung cancer," Annals of Oncology, vol. 22, no. 2, pp. 383-389, 2011.

[42] M. Chatterjee, S. Mohapatra, A. Ionan et al., "Diagnostic markers of ovarian cancer by high-throughput antigen cloning and detection on arrays," Cancer Research, vol. 66, no. 2, pp. 1181-1190, 2006.

[43] X. Wang, J. Yu, A. Sreekumar et al., "Autoantibody signatures in prostate cancer," The New England Journal of Medicine, vol. 353, no. 12, pp. 1224-1235, 2005.

[44] D. Lu, E. Kuhn, R. E. Bristow et al., "Comparison of candidate serologic markers for type I and type II ovarian cancer," Gynecologic Oncology, vol. 122, no. 3, pp. 560-566, 2011.

[45] G. Yahalom, D. Weiss, I. Novikov et al., "An antibody-based blood test utilizing a panel of biomarkers as a new method for improved breast cancer diagnosis," Biomarkers in Cancer. In press. 


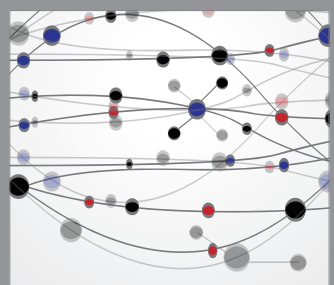

The Scientific World Journal
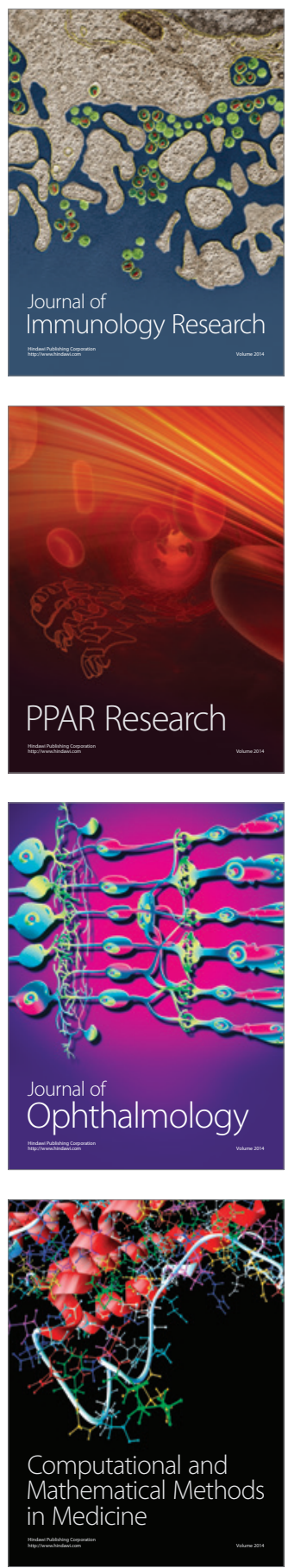

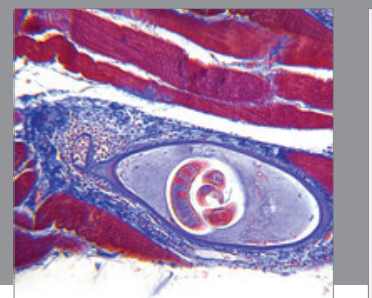

Gastroenterology

Research and Practice
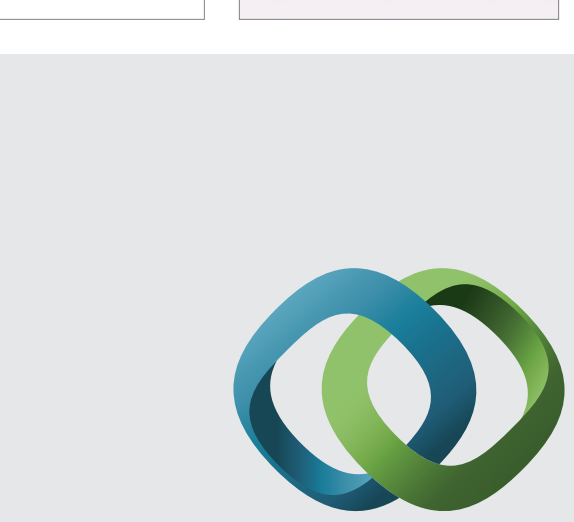

\section{Hindawi}

Submit your manuscripts at

http://www.hindawi.com
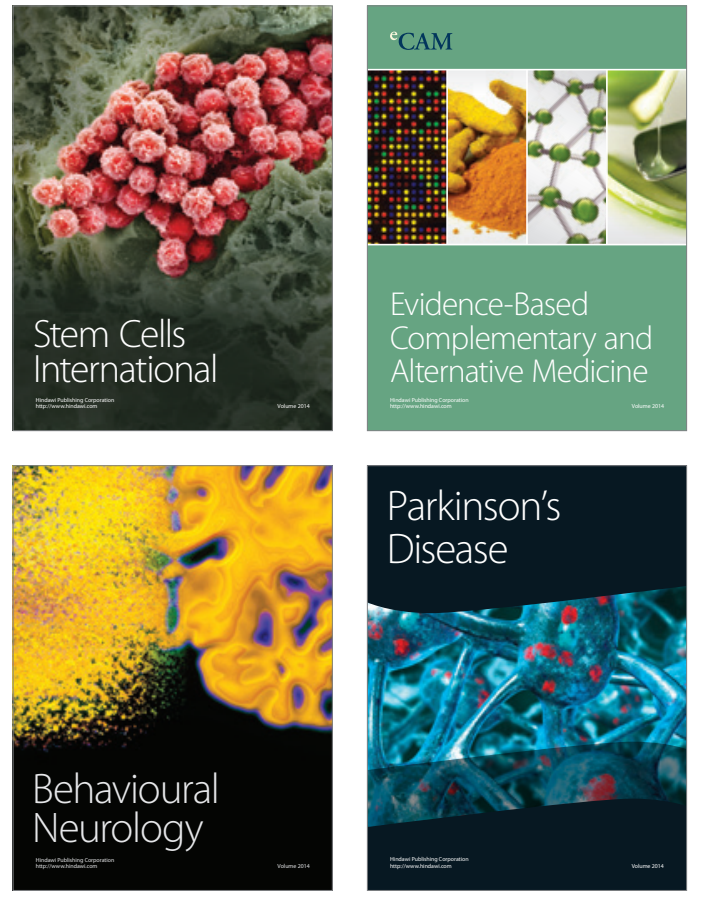
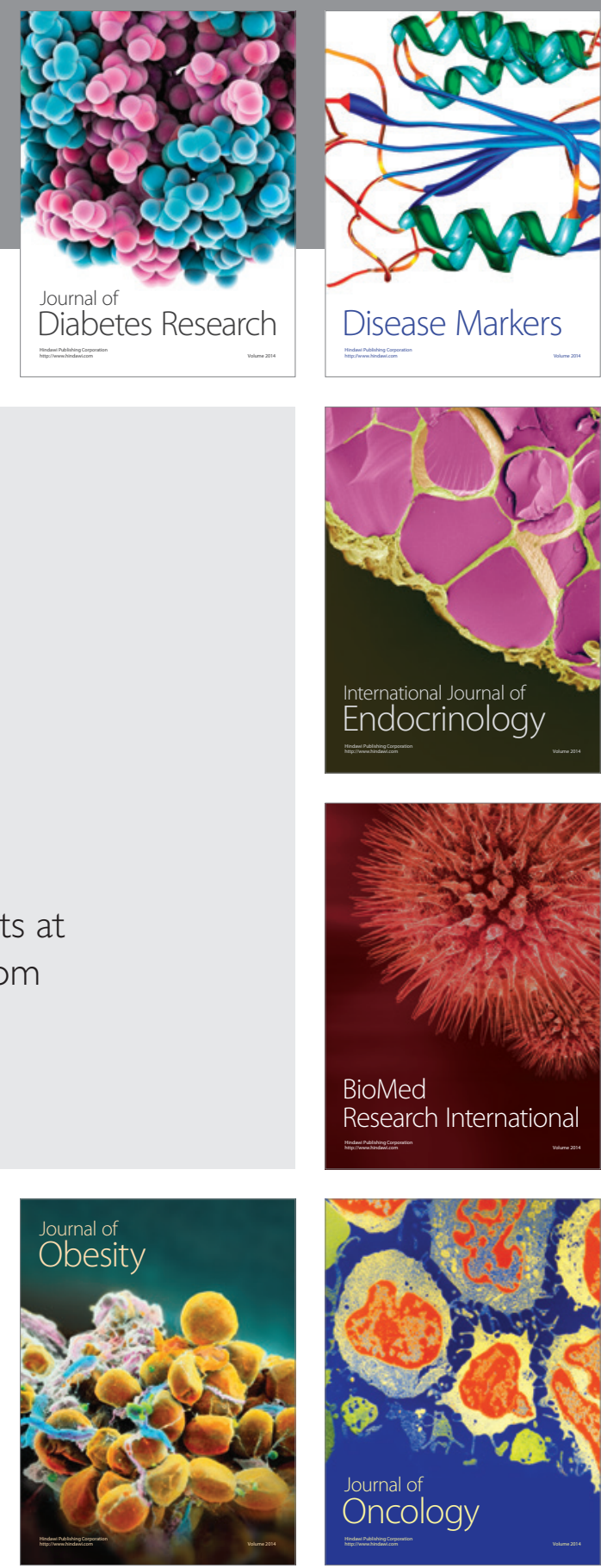

Disease Markers
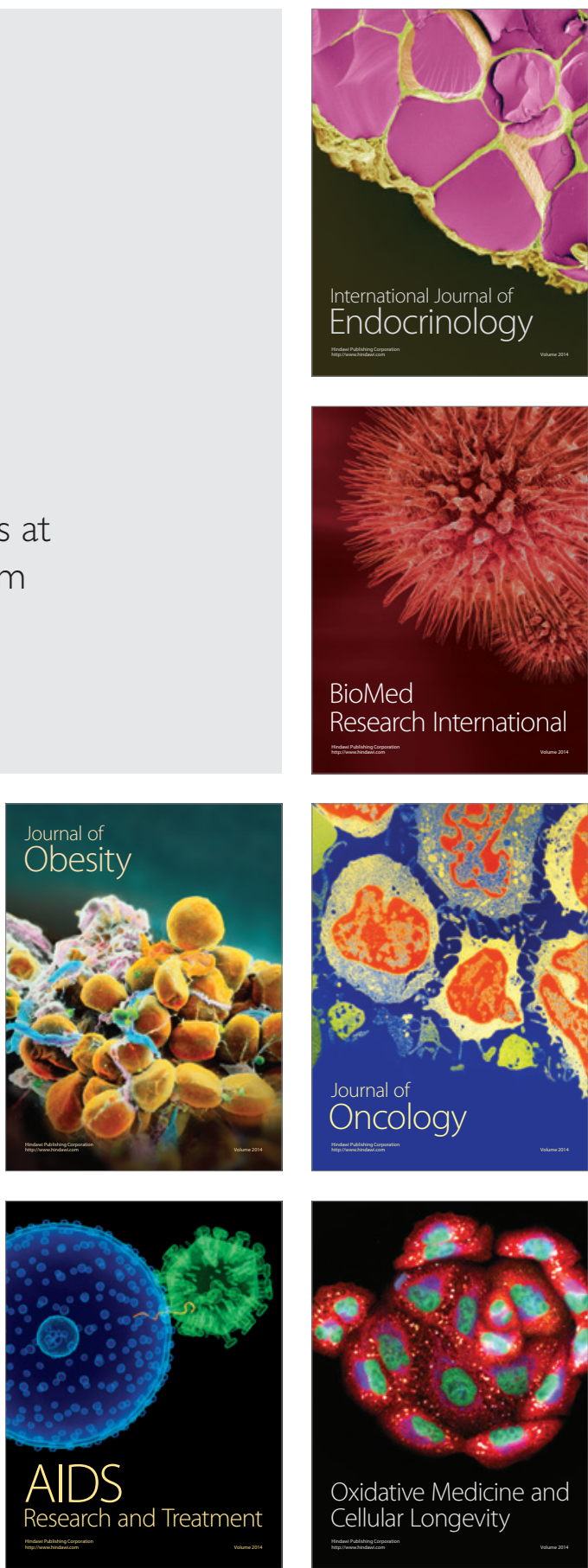